\title{
SUBMERSIVE AND UNIPOTENT GROUP QUOTIENTS AMONG SCHEMES OF A COUNTABLE TYPE OVER A FIELD $k$
}

BY

\section{PAUL CHERENACK}

ABSTRACT. An algebraic group $G$ is called submersive if every quotient in affine schemes $c^{G}: \operatorname{Spec} A \rightarrow \operatorname{Spec} A^{G}$ which is surjective is also submersive. We prove that every unipotent group is submersive. Suppose $G$ is submersive. We show that if $c^{G}(\operatorname{Spec} A)$ is open in Spec $A^{G}$ or if some restrictions on the action of $G$ on $A$ are made, $c^{G}$ is a topological quotient. $A$ criterion for semisimplicity of points is extended to the case where $G$ is unipotent. Finally, applications of the theory are provided.

0 . Introduction. Let $X$ be an affine scheme of finite type over a field $k$ and suppose that $G$ is an irreducible algebraic group over $k$ which has a closed action on $X$ via a $k$ morphism $\sigma: G \times X \rightarrow X$. If $X=\operatorname{Spec} A, A^{G}$ consists of the functions in $A$ invariant under $G$ and $X^{G}=\operatorname{Spec} A^{G}$, we call the map $c^{G}: X \rightarrow X^{G}$ induced by the inclusion $A^{G} \rightarrow A$ the algebraic group quotient of $X$ by $G$. It is easily seen that if $G$ is affine, the mapping $c^{G}$ is the coequalizer of $\sigma$ and $P_{2}$, the projection of $G \times X$ onto $X$, in the category of affine schemes of a countable type over $k$ (see [2]).

Definition 1. Let $X$ be a free variable. If $c^{G}: X \rightarrow X^{G}$ is surjective implies that $c^{G}: X \rightarrow X^{G}$ is submersive, we say that $G$ is submersive.

Every reductive group is submersive. See Mumford [6, p. 27, Theorem 1.1]. In $\$ 4$, we show that every unipotent group is submersive.

The basic notation to be used here is that of Mumford [6]. However, we present now some of the conceptions to be found in Mumford [6] in a more compact form using some category theory.

Definition 2. Let $D_{1}$ be a subcategory of $D_{2}$ and $D_{2}$ be a subcategory of $D_{3}$ (thus, $D_{1}, D_{2}$ and $D_{3}$ are categories). Suppose that $f, g: Y_{1} \rightrightarrows Y_{2}$

Received by the editors May 10, 1974 .

AMS (MOS) subject classifications (1970). Primary 14A15, 14D20, $14 \mathrm{C} 10$.

Key words and phrases. Submersive group, unipotent group, scheme of a countable type over $k$, affine scheme, algebraic group quotient, reductive group, relative coequalizer, semistable point, Hilbert's fourteenth problem, Nagata's counterexample, linear systems, Chevalley's theorem, constructible set. 
are two maps in $D_{1}$. A $D_{3}$ coequalizer in $D_{2}$ relative to $D_{1}$ is a map $c: Y_{2} \rightarrow Y_{3}$ in $D_{2}$ such that, for each map $b: Y_{2} \rightarrow Y_{4}$ in $D_{3}$ satisfying $b \circ f=b \circ g$, there is a unique map $i: Y_{3} \rightarrow Y_{4}$ in $D_{3}$ with $i \circ c=b$.

The most important theorem of this paper is the theorem that follows. In the paper $A^{G} \not k$.

Theorem 1. Let $X$ and $X^{G}$ be those affine schemes and $G$ be that algebraic group considered above. Assume that $G$ is submersive and affine. Then, under each of the following conditions:

(i) $c^{G}(X)$ is open in $X^{G}$,

(ii) for each $h \in A$ and each $\sigma \in G$, there is a sequence of functions $b_{1}, \ldots, h_{n}$ in $A$ such that

$$
h^{\sigma}=h+h_{1}, \quad h_{1}^{\sigma}=h_{1}+h_{2}, \ldots, h_{n-1}^{\sigma}=h_{n-1}+h_{n}
$$

with $h_{n} \in A^{G}, c^{G}: X \rightarrow c^{G}(X)$ is a Top and Sch coequalizer of the maps

$$
\sigma, P_{2}: G \times X \rightrightarrows X
$$

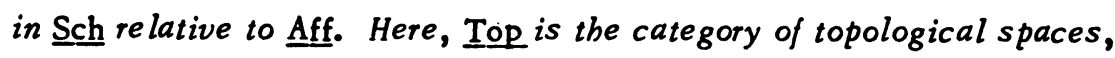
Sch is the category of schemes and Aff is the category of affine schemes.

In $\$ 2$ we show that condition (ii) of Theorem 1 implies condition (i). Then, in $\$ 6$, we provide some examples where condition (ii) is satisfied.

Corollary 1. Let $X$ and $X^{G}$ be those affine schemes and $G$ be that algebraic group of Theorem 1. Suppose that $G$ is a unipotent group. Then, $c^{G}: X \rightarrow c^{G}(X)$ is a Top and Sch coequalizer of $\sigma$ and $P_{2}$ in $\underline{\text { Sch relative to }}$ Aff.

Proof. In $\$ 4$, we show that every unipotent group is submersive. Apply Theorem 1.

Corollary 2. Let $X$ and $X^{G}$ be those affine schemes and $G$ be that algebraic group of Theorem 1. Suppose that $c^{G}(X)$ is of finite type over $k$ and that $k$ is an algebraically closed field. If $X$ is irreducible and normal and if the residue field of the generic point of $c^{G}(X)$ has characteristic 0 , then $c^{G}: X \rightarrow c^{G}(X)$ is a geometric quotient of $X$ by $G$, i.e., the mapping $c^{G}$ is

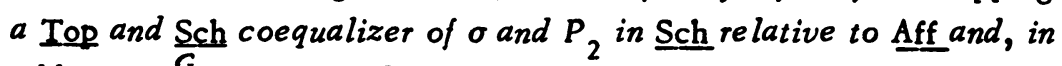
addition, $c^{G}$ is universally submersive.

Proof. One applies Mumford [6, Proposition 0.2, p. 7].

Remark 1. Mumford [6, p. 6] indicates that if $X$ is normal over $k$ and $c^{G}: X \rightarrow c^{G}(X)$ is a geometric quotient, then $c^{G}(X)$ must be of finite type 
over $k$. Nagata's example (see Dieudonné [4, p. 45]) shows us that $X$ can be of finite type and normal but that $X^{G}$ may not be of finite type. $A$ simple example illustrates, however, that $c^{G}(X)$ could be of finite type over $k$ even though it is an open subscheme of an affine scheme of countable type over $k$. Let $i, *: k^{1} \rightrightarrows k^{2}$ be two maps where $i$ is a closed immersion identifying $k^{1}$ with $X=0,(0,0) \in i\left(k^{1}\right)$ and $*\left(k^{1}\right)=(0,0)$. Then, $i$ and $*$ have a coequalizer $k^{2} / k^{1}=\operatorname{Spec}(k+(X))$ in Sch where $(X)$ is the ideal generated by $X$ in $k[X, Y]$. Furthermore, $k+(X)$ is not of finite type over $k$; see [2]. Localize at $X \neq 0$. Then, $(k+(X))_{X} \simeq k[X, Y]_{X}$. Let $q: k^{2} \rightarrow k^{2} / k^{1}$ be the quotient map. It follows that $k^{2 / k^{1}}-q\left(k^{1}\right) \simeq k^{2}-k^{1}$ is of finite type over $k$. This suggests the following refinement of

Hilbert's Fourteenth Problem. Is $c^{G}(X)$ a scheme of finite type over $k$ ?

In order to provide some applications of Theorem 1, we will prove the next result in $\$ 5$.

Theorem 2. Let $G$ be a unipotent affine algebraic group which acts on projective space $\mathrm{P}^{n}$ by a closed action over $k$. Consider the cone $k^{n+1}$ lying above $\mathbf{P}^{n}$. If $x$ is a point of $\mathbf{P}^{n}$, suppose that $x^{\prime}$ denotes a point lying above $x$. Note that the action of $G$ on $\mathrm{P}^{n}$ extends to an action of $G$ on $k^{n+1}$. Then, $x$ is semistable if and only if the closure of the orbit of $x^{\prime}$ does not contain 0 .

For further information on the notions contained in this theorem, the reader is referred to Mumford [6, p. 50].

Examples of unipotent actions on projective spaces, which appear as linear systems, will be provided in $\$ 6$.

1. A partial generalization of Chevalley's theorem.

Lemma 1. Let $f: X \rightarrow Y$ be a dominant morphism of affine schemes defined over $k$ and suppose that $X$ is of finite type over $k$. Then, $f(X)$ contains an open subset $U$ of $Y$.

Proof. Clearly, we can assume that $X$ and $Y$ are reduced. Suppose that $X_{1}, X_{2}, \ldots, X_{m}$ are the components of $X$ (finite in number as $X$ is of finite type). Consider the mapping $f_{i}: X_{i} \rightarrow \overline{f\left(X_{i}\right)}$, the restriction of $f$ to $X_{i}$ mapped to the closure of $f\left(X_{i}\right), i=1,2, \ldots, m$. If $f\left(X_{i}\right)$ contains an open subset $U_{i}$ of $\overline{f\left(X_{i}\right)}$, then $f\left(X_{i}\right)$ also contains an affine open subset $U\left(g_{i}\right)$ of $\overline{f\left(X_{i}\right)}$, the complement of the hyperplane $g_{i}$. In that case, $f(X)=$ 
$\bigcup_{i=1}^{m} f\left(X_{i}\right)$ contains an affine open $U\left(g_{1} g_{2} \cdots g_{m}\right)$ of $\overline{f(X)}=\bigcup_{i=1}^{m} \overline{f\left(X_{i}\right)}$. Therefore, we can assume in Lemma 1 that $X$ and $Y$ are irreducible and reduced.

The remainder of the proof, modulo some minor modifications, can be found in Mumford [7, Pp. 94 and 95].

Remark. When $Y$ is of finite type, Lemma 1 follows from Chevalley's theorem which asserts (see Grothendieck [s]) that $f(X)$ is constructible.

2. $c^{G}(X)$ is open in $X^{G}$. We employ the notation of $\$ 0$. In order to show that $c^{G}: X \rightarrow c^{G}(X)$ is a Top coequalizer in $\underline{\mathrm{Sch}}$, we must show that $c^{G}(X)$ is a scheme. This result is a consequence of the next lemma.

Lemma 2. If condition (ii) of Theorem 1 holds, $c^{G}(X)$ is open in $X^{G}$.

Proof. Lemma 1 implies that there is an affine open subset $U\left(g_{1}\right)$ of $X^{G}$ such that $U\left(g_{1}\right) \subset c^{G}(X) \subset X^{G}$. If $c^{G}(X)=X^{G}$ or $c^{G}(X)=U\left(g_{1}\right)$, we are finished. Suppose, then, that $c^{G}(X) \neq X^{G}$ and $c^{G}(X) \neq U\left(g_{1}\right)$ and consider the map

$$
c_{1}^{G}: \operatorname{Spec} A /\left(g_{1}\right) \rightarrow \operatorname{Spec} A^{G} /\left(g_{1}\right)
$$

where $\left(g_{1}\right)$ denotes the ideal generated by $g_{1}$ in $A$ and $A^{G}$, respectively.

Suppose that $c_{1}^{G}$ is dominant. Lemma 1 implies that there is an open subset $U\left(g_{2}\right)$ of $\operatorname{Spec} A G /\left(g_{1}\right)$ such that

$$
U\left(g_{2}\right) \subset c_{1}^{G}\left(\operatorname{Spec} A /\left(g_{1}\right)\right) \subset \operatorname{Spec} A^{G} /\left(g_{1}\right) .
$$

If (a) $U\left(g_{2}\right)=c_{1}^{G}\left(\operatorname{Spec} A /\left(g_{1}\right)\right)$ or $\left(b_{1} c_{1}^{G}\left(\operatorname{Spec} A /\left(g_{1}\right)\right)=\operatorname{Spec} A G /\left(g_{1}\right)\right.$, we are finished. In case $(a)_{1}, c^{G}(X)=X^{G}-\left(V\left(g_{1}\right) \cap V\left(g_{2}\right)\right)$ (where $V(g)$ denotes the hypersurface defined by $g$ in $X^{G}$ ) and hence $c^{G}(X)$ is open in $X^{G}$. In case $(b)_{1}, c^{G}(X)=X^{G}$. This contradicts a previous assumption. Assume therefore that $(a)_{1}$ and $(b)_{1}$ do not hold.

If $A$ is an integral domain, $g_{1} \in A^{G}$ and $x=a g_{1} \in A^{G}$, then $a \in A^{G}$. In this case, one sees easily that $A G /\left(g_{1}\right) \rightarrow A /\left(g_{1}\right)$ is injective and hence $c_{1}^{G}$ is dominant.

Claim (n). If $g_{1}, \ldots, g_{n} \in A^{G}$, then

$$
c^{G}: \operatorname{Spec} A^{G} /\left(g_{1}, \ldots, g_{n}\right) \rightarrow \operatorname{Spec} A /\left(g_{1}, \ldots, g_{n}\right)
$$

is injective.

Proof of Claim (n). Suppose that $g_{1}, g_{2}, \ldots, g_{n} \in A^{G}$ and 
$(y)$

$$
a_{1} g_{1}+a_{2} g_{2}+\cdots+a_{n} g_{n}=0
$$

with $a_{1}, a_{2}, \ldots, a_{n} \in A$. We show that there are $a_{1}^{\prime}, a_{2}^{\prime}, \ldots, a_{n}^{\prime} \in A^{G}$, not all zero, such that $a_{1}^{\prime} g_{1}+a_{2}^{\prime} g_{2}+\cdots+a_{n}^{\prime} g_{n}=0$.

$$
\text { If } f=a_{1} g_{1}+a_{2} g_{2}+\cdots+a_{n-1} g_{n-1} \in A^{G} \text {, taking } a_{n}=-1 \text { and } g_{n}=f
$$
above, $f=a_{1}^{\prime} g_{1}+\cdots+a_{n-1}^{\prime} g_{n}$ for elements $a_{1}^{\prime}, \ldots, a_{n-1}^{\prime} \in A^{G}$. This implies that the map $A G /\left(g_{1}, \ldots, g_{n-1}\right) \rightarrow A /\left(g_{1}, \ldots, g_{n-1}\right)$ is injective and proves the claim.

Suppose that $a_{1} \notin A^{G}$. There is then a $\sigma \in G$ such that $a_{1}^{\sigma} \neq a_{1}$. Condition (ii), Theorem 1, can be applied to determine infinite sequences $a_{j}^{i}$, $i=0,1, \ldots ; j=1, \ldots, n$, of functions such that, for $j=1, \ldots, n,\left(a_{j}^{i}\right)^{\sigma}=$ $a_{j}^{i}+a_{j}^{i+1}$ where $a_{j}=a_{j}^{0}$ and, for some integer $m(j), a_{j}^{m(j)}$ is in $A^{G}$. Note that $a_{j}^{m(j)+1}=0$. We can assume that $a_{j}^{i}, i<m(j)$, is not invariant under $\sigma$ and that $m=\max (m(j))=m(1)$.

Applying $\sigma$ to $\gamma$, we find that

$$
\left(a_{1}+a_{1}^{1}\right) g_{1}+\left(a_{2}+a_{2}^{1}\right) g_{2}+\cdots+\left(a_{n}+a_{n}^{1}\right) g_{n}=0
$$

or using $\gamma$ that

$$
a_{1}^{1} g_{1}+a_{2}^{1} g_{2}+\cdots+a_{n}^{1} g_{n}=0 .
$$

This process can be used $m$ times to show that

$$
a_{1}^{m} g_{1}+a_{2}^{m} g_{2}+\cdots+a_{n}^{m} g_{n}=0 .
$$

Since $a_{1}^{m-1}$ is not invariant under $\sigma, a_{1}^{m} \neq 0$. Clearly, $a_{1}^{m}, a_{2}^{m}, \ldots, a_{n}^{m} \epsilon$ $A^{G}$. The claim is proven.

Continuing as we have, we obtain at stage $n$ an affine open subset $U\left(g_{n}\right)$ of Spec $A^{G} /\left(g_{1}, g_{2}, \ldots, g_{n-1}\right)$ from a dominant map $c_{n-1}^{G}$ such that $U\left(g_{n}\right) \subset c_{n-1}^{G}\left(\operatorname{Spec} A /\left(g_{1}, g_{2}, \ldots, g_{n-1}\right)\right) \subset \operatorname{Spec} A^{G} /\left(g_{1}, \ldots, g_{n-1}\right)$. If

(a) $U\left(g_{n}\right)=c_{n-1}^{G}\left(\operatorname{Spec} A /\left(g_{1}, \ldots, g_{n-1}\right)\right)$, or

(b) $c_{n-1}^{G}\left(\operatorname{Spec} A /\left(g_{1}, \ldots, g_{n-1}\right)\right)=\operatorname{Spec} A^{G} /\left(g_{1}, \ldots, g_{n-1}\right)$, we are done. In case $(a)_{n}, c^{G}(X)=X^{G}-\left(\bigcap_{i=1}^{n} V\left(g_{i}\right)\right)$. In case (b) $)_{n}$ is true, we find that $(b)_{n-1}$ is true. Assume that $(a)_{n}$ and $(b)_{n}$ are not true.

Let

$$
x_{n}=\left(c^{G}\right)^{-1}\left(x^{G}-\left(\bigcap_{i=1}^{n} V\left(g_{i}\right)\right)\right) .
$$


Then, $X_{n}$ is an open subset of $X$; and because of the nature of our construction, i.e. we assume (b) $)_{n}$ is not true, if $X \neq X_{n}$, then $X_{n} \subsetneq X_{n+1} \cdot$ But, as $X$ is of finite type over $k$ and hence satisfies the ascending chain condition on open sets, there is an integer $N$ such that $X_{N}=X$. It follows that

$$
{ }^{G}(X)=c^{G}\left(X_{N}\right)=X^{G}-\left(\bigcap_{i=1}^{N} V\left(g_{i}\right)\right) \text { Q.E.D. }
$$

3. Proof of Theorem 1. According to Mumford [6, p. 8], a categorical quotient $\left(c^{G}(X), c^{G}\right)$ to $\sigma$ and $P_{2}$ exists when the following conditions hold:

(i) $c^{G} \circ \sigma=c^{G} \circ P_{2}$.

(ii) ${ }_{-}^{o} G(X)$ is the sheaf of invariants of $\left.c_{*}^{G}\left(\varrho_{X}\right)\right|_{c}{ }{ }_{(X)}$.

(iii) If $W$ is an invariant closed subset of $X, c^{G}(W)$ is closed in $X^{G}$; if $\left\{W_{i}\right\}, i \in I$, form a set of invariant closed subsets of $X$, then

$$
c^{G}\left(\bigcap_{i \in I} W_{i}\right)=\bigcap_{i \in I} c^{G}\left(W_{i}\right)
$$

Let $\left\{U_{j}\right\}_{j \in J}$ be a covering of $c^{G}(X)$ by affine opens. We employ the proposition below demonstrated in [3].

Proposition 1. In the category of countably generated $k$ algebras, localization preserves equalizer.

As a consequence of this proposition, the maps

$$
c_{j}^{G}:\left(c^{G}\right)^{-1}\left(U_{j}\right) \rightarrow U_{j} \quad(j \in J)
$$

are algebraic quotients of $\left(c^{G}\right)^{-1}\left(U_{j}\right)$ by $G$ and surjective. Furthermore; it is not difficult to see that if (i), (ii) and (iii) hold for $c_{j}^{G}$ and $c_{j}^{G}$ is a topological group quotient, $j \in J$, (i), (ii) and (iii) hold for $c^{G}$ and $c^{G}$ is a topological group quotient. Therefore, we can assume that $c^{G}(X)=X^{G}$.

Having made the last assumption, (i) is obvious. (ii) follows from Proposition 1 and the fact that the defining property of a sheaf is an equalizing diagram. As $G$ is submersive, (iii) will follow from the next proposition. See Mumford [6, p. 28].

Proposition 2. If the mapping $c^{G}: X \rightarrow X^{G}$ is surjective and submersive and if $A_{i}, i \in I$, are the invariant (reduced) ideals in A corresponding to the $W_{i}$ in (iii), then there is an equality 


$$
\left(\sum_{i \in I} A_{i}\right)^{1 / 2} \cap A^{G}=\left(\sum_{i \in I}\left(A_{i} \cap A^{G}\right)\right)^{1 / 2}
$$

Proof of Proposition 2. We have

$$
\left(\sum_{i \in I} A_{i}\right)^{1 / 2} \cap A^{G}=\left(\sum_{i \in I}\left(\bigcap_{p \in W_{i}} p\right)\right)^{1 / 2} \cap A^{G}=\left(\bigcap_{p \in \cap w_{i}} p\right) \cap A^{G} .
$$

As $c^{G}$ is submersive and surjective,

$$
A_{i} \cap A^{G}=\bigcap_{p \in W_{i}}\left(p \cap A^{G}\right)
$$

Therefore,

$$
\left(\sum_{i \in I}\left(a_{i} \cap A^{G}\right)\right)^{1 / 2}=\left(\sum_{i \in I}\left(\bigcap_{p \in W_{i}}\left(p \cap A^{G}\right)\right)\right)^{1 / 2}=\bigcap_{p \in \cap W_{i}}\left(p \cap A^{G}\right)
$$

The first and last equalities imply the proposition. Note that the intersection in (*) contains all the prime ideals of $A^{G}$ containing $A_{i} \cap A^{G}$. Otherwise, we cannot conclude the last equality.

To demonstrate that $c^{G}$ is a topological quotient and, thus, to complete the proof of Theorem 1, we must show that under the action of $G$, distinct orbits are mapped by $c^{G}$ into distinct points. The action of $G$ on $X$ is closed. Let $A_{1}$ and $A_{2}$ be the defining ideals of two distinct orbits $X_{1}$ and $X_{2}$ of $X$ under $G$. Then

$$
A^{G}=\left(A_{1}+A_{2}\right)^{1 / 2} \cap A^{G}=\left(\left(A_{1} \cap A^{G}\right)+\left(A_{2} \cap A^{G}\right)\right)^{1 / 2} \text {. }
$$

Hence, $1=f+g$, where $f, g \in A^{G}, f$ vanishes on $c^{G}\left(X_{1}\right)$ and $g$ vanishes on $c^{G}\left(X_{2}\right)$. It must be the case, then, that $c^{G}\left(X_{1}\right) \neq c^{G}\left(X_{2}\right)$. Q.E.D.

4. Unipotent groups are submersive. Note that every representation of a unipotent group is through a unipotent action. See Borel [1, p. 151]. The fact that unipotent groups are submersive follows, then, immediately from the next proposition.

Proposition 3. Let $G$ be an algebraic group such that every finite dimensional representation of $G$ has at least one fixed point. Then $G$ is submersive.

Proof. Suppose that $c^{G}: X \rightarrow X^{G}$ is surjective where $X=\operatorname{Spec} A$ and $X^{G}=\operatorname{Spec} A^{G}$. Consider an invariant closed set $W$ of $X$ and let $J$ be an 
invariant ideal whose zeroes equal $W . J$ is the union of finite dimensional $G$ invariant subspaces. See Mumford [6, p. 25]. As every finite dimensional representation of $G$ has at least one fixed point, clearly $J$ contains an element $g_{1}$ of $A^{G}$.

Claim. $c_{1}^{G}:$ Spec $A /\left(g_{1}\right) \rightarrow \operatorname{Spec} A^{G} /\left(g_{1}\right)$ is surjective.

Proof of claim. If $q \in \operatorname{Spec} A^{G} /\left(g_{1}\right), q$ has the form $p \cap A^{G}$ as $c^{G}$ is surjective. As $q$ contains $g_{1}$, so does $p$. Therefore, $p \in \operatorname{Spec} A /\left(g_{1}\right)$.

Repeating this argument with $c_{1}^{G}$ instead of $c^{G}$ and $J /\left(g_{1}\right)$ instead of $J$, we obtain a surjective map $c_{2}^{G}$ : Spec $A /\left(g_{1}, g_{2}\right) \rightarrow \operatorname{Spec} A G /\left(g_{1}, g_{2}\right)$ where $g_{2} \in J$ but $g_{2} \notin\left(g_{1}\right)$.

Continuing with this process, at stage $n$, we obtain a surjective map

$$
c_{n}^{G}: \operatorname{Spec} A /\left(g_{1}, g_{2}, \ldots, g_{n}\right) \rightarrow \operatorname{Spec} A^{G} /\left(g_{1}, g_{2}, \ldots, g_{n}\right)
$$

where $\left(g_{1}, g_{2}, \ldots, g_{n}\right) \subset J$ but $g_{i} \notin\left(g_{1}, g_{2}, \ldots, g_{i-1}\right)$. As $X$ is of finite type over $k$, there is an integer $N$ such that $J=\left(g_{1}, g_{2}, \ldots, g_{N}\right)$.

$$
c_{N}^{G}: \operatorname{Spec} A /\left(g_{1}, g_{2}, \ldots, g_{N}\right) \rightarrow \operatorname{Spec} A^{G} /\left(g_{1}, g_{2}, \ldots, g_{N}\right)
$$

is surjective. As $W=\operatorname{Spec} A / J$ and $\operatorname{Spec} A^{G} /\left(g_{1}, g_{2}, \ldots, g_{N}\right)$ is closed in $X^{G}, c^{G}(W)$ is a closed subset of $X^{G}$. Q.E.D.

5. Proof of Theorem 2. The direction: $x$ is semistable implies that 0 is not in the closure of the orbit $O\left(x^{\prime}\right)$ of $x^{\prime}$ is clear. See Mumford [6, p. 50, Proposition 2.2].

Suppose that 0 is not in the closure of the orbit $O\left(x^{\prime}\right)$ of $x^{\prime}$. Let $W_{1}$ be the closure of $O\left(x^{\prime}\right)$ and $W_{2}$ be $0 . W_{1}$ and $W_{2}$ are disjoint invariant closed subsets of $K^{n+1}$. To prove that $x$ is semistable and hence to prove Theorem 2, we need to show that for some positive integer $m$, there is an $F \in H^{0}\left(\mathrm{P}^{n}, \vartheta_{\mathrm{p} n}(m)\right)$ such that $F(x) \neq 0$. First, we consider the affine situation where the following lemma is needed.

Lemma 3. Suppose that the map $c^{G}: X \rightarrow X^{G}$ is submersive. If $X_{1}$ and $X_{2}$ are two disjoint invariant closed subschemes of $X$, there is an element $f \in \Gamma\left(X^{G}, \vartheta_{X} G\right)$ which is 1 on $X_{1}$ and 0 on $X_{2}$.

Proof. As in the proof of Theorem 1 , as $c^{G}$ is submersive, applying Proposition 2, one arrives at the equation

$$
A^{G}=\left(A_{1}+A_{2}\right)^{1 / 2} \cap A^{G}=\left(\left(A_{1} \cap A^{G}\right)+\left(A_{2} \cap A^{G}\right)\right)^{1 / 2}
$$


where $A_{1}$ and $A_{2}$ are the defining ideals of $X_{1}$ and $X_{2}$, respectively. Then $1=f+g$ where $f$ vanishes on $X_{2}, g$ vanishes on $X_{1}$ and $f, g \in A^{G}$. Q.E.D.

Now, as we have seen in the proof of Theorem 1 , the map $c^{G}$ restricts to mappings of affine opens $c_{j}^{G}:\left(c^{G}\right)^{-1}\left(U_{j}\right) \rightarrow U_{j}$ where $\left\{U_{j}\right\}_{j \in J}$ is an open affine covering of $c^{G}(X)$. We examine such maps in our present situation, i.e., $X=k^{n+1}$ and $G$ is a unipotent group acting on $X$. Also, we place $V_{j}=$ $\left(c^{G}\right)^{-1}\left(U_{j}\right)$.

Let $V_{j}$ be the complement of the hypersurface $g_{j}$. As $k^{n+1}$ is of affine type over $k$, only a finite number of $V_{j}, j=1, \ldots, m$, are needed to cover $k^{n+1}$. Pick a point $Q \in W_{1}$. By taking an appropriate linear combination of the $g_{j}, j=1, \ldots, m, g_{0}=d_{1} g_{1}+d_{2} g_{2}+\cdots+d_{m} g_{m}$, one can guarantee that $0, Q \in V_{0}$ where $V_{0}$ is the complement of the hypersurface $g_{0}$. Thus, also, $W_{1} \cap V_{0} \neq 0$. Proposition 3 implies, as $G$ is unipotent, that $c_{0}^{G}: V_{0}=\left(c^{G}\right)^{-1}\left(U_{0}\right) \rightarrow U_{0}$ is submersive. By Lemma 3 , there is a function $f$ on $V_{0}$ which is one on $W_{1} \cap V_{0}$ and such that $f(0)=0$. $f$ has the form $h /\left(g_{0}\right)^{m} j$ where $h \in k\left[X_{0}, X_{1}, \ldots, X_{n}\right], b$ does not vanish on $w_{1} \cap V_{0}$ and $h(0)=0$. $b$ is an invariant function as $f$ and $g_{0}$ are invariant functions. $b$ is, thus, constant on $O\left(x^{\prime}\right)$. But, then, $b$ must be constant nonzero on $W_{1}$. Write $b=b_{l}+\cdots+b_{l+r}$ where $h_{i}$ is homogeneous of degree $i$. As $b(0)=0$, $l \geq 1$. For some $i, l \leq i \leq l+r, b_{i}$ does not vanish at $x^{\prime}$. Let $F$ be that $h_{i}$. Then, $F$ is an invariant homogeneous polynomial such that $F(x) \neq 0$. Q.E.D.

6. Examples of quotients by unipotent actions. In this section $k$ is the field of complex numbers.

Example 1. Let $U$ be the unipotent group which acts on $k^{2}$ and consists of matrices of the form $\left(\begin{array}{ll}1 & t \\ 0 & 1\end{array}\right)$. The action of $U$ on $k^{2}$ induces an action of $U$ on lines of the form $a_{0}+a_{1} X+a_{2} Y=0$ in $k^{2}$ where $a_{2} \neq 0$. Under this action, lines which pass through a fixed point on the $Y$ axis (other than $X=0$ ) are identified. Thus, as it is easy to verify $X^{U}=c^{U}(X)=k^{1}$ where $X=k^{2}$ represents the collection of lines where $a_{2} \neq 0$. Extending the action of $U$ to $\mathrm{P}^{2}$, one sees that $X^{U}=\mathrm{P}^{1}$.

Example 2. Let $H$ be a hyperplane in $\mathbf{P}^{2}$ and consider the operation of PGL(2) on $|m H|$. See Mumford [6, p. 79]. The action of PGL(2) induces an action of the unipotent subgroup $U$ of SL(3) whose elements are of the form 


$$
\left(\begin{array}{lll}
1 & 0 & t \\
0 & 1 & 0 \\
0 & 0 & 1
\end{array}\right)
$$

on $|m H|$. Suppose that $m=4$. Then $|4 H|$ consists of polynomials of the form

$$
\begin{aligned}
& a_{40} X_{1}^{4}+a_{31} X_{1}^{3}+a_{22} X_{1}^{2} X_{1}^{2}+a_{13} X_{1} X_{2}^{3}+a_{04} X_{2}^{4} \\
& +a_{30} X_{0} X_{1}^{3}+a_{21} X_{0} X_{1}^{2} X_{2}+a_{12} X_{0} X_{1} X_{2}^{2}+a_{03} X_{0} X_{2}^{3} \\
& +a_{20} X_{0}^{2} X_{1}^{2}+a_{11} X_{0}^{2} X_{1} X_{2}+a_{02} X_{0}^{2} X_{2}^{2} \\
& +a_{10} X_{0}^{3} X_{1}+a_{00} X_{0}^{4}+a_{01} X_{0}^{3} X_{2} .
\end{aligned}
$$

The action of $U$ on $|4 H|$ sends $\left(a_{i j}\right)$ onto

$$
\begin{gathered}
\left(a_{40}, a_{31}, a_{22}, a_{13}, a_{04}, a_{30}+t a_{31}, a_{21}+2 t a_{22}, a_{12}+3 t a_{13}, a_{03}+4 t a_{04},\right. \\
a_{20}+t^{2} a_{22}+t a_{21}, a_{11}+3 t^{2} a_{13}+2 t a_{12}, a_{02}+3 t a_{03}+6 t^{2} a_{04} \\
a_{10}+t^{3} a_{13}+t^{2} a_{12}+t a_{11}, a_{01}+4 t^{3} a_{04}+3 t^{2} a_{03}+2 t a_{02}, \\
\left.a_{01}+t^{4} a_{04}+t^{3} a_{03}+t^{2} a_{02}+t a_{01}\right) .
\end{gathered}
$$

We apply Theorem 2. It follows from the above representation of the action of $U$ that the orbit of $\left(a_{i j}\right)$ as a point of $k^{15}$ does not have 0 as a limit point and that condition (ii) of Theorem 1 is satisfied. Therefore, employing Theorem 1 , there is a quotient of $|4 H|$ by $U$ and this quotient is topological. See $[6$, p. 38].

Note that the quotient of $|4 H|$ by SL(3) may not exist. See Mumford $[6$, p. 80]. The chief problem in taking a unipotent quotient is that little is known about the interpretation of unipotent quotients.

Example 3. Suppose that we are in the same situation as in Example 2 except let $U$ now be the full unipotent subgroup of SL(3), i.e., $U$ consists of all matrices of the form

$$
\left(\begin{array}{lll}
1 & w & t \\
0 & 1 & u \\
0 & 0 & 1
\end{array}\right)
$$

The action of $U$, in this case, on $|4 H|$ sends $\left(a_{i j}\right)$ into $\left(a_{i j}^{\prime}\right)$ where 


$$
\begin{aligned}
& a_{40}^{\prime}=a_{40}+u a_{31}+u^{2} a_{22}+u^{3} a_{13}+u^{4} a_{04} \\
& a_{31}^{\prime}=a_{31}+2 u a_{22}+3 u^{2} a_{13}+4 u^{3} a_{04} \\
& a_{22}^{\prime}=a_{22}+3 u a_{13}+6 u^{2} a_{04} \\
& a_{13}^{\prime}=a_{13}+4 u a_{04} \\
& a_{04}^{\prime}=a_{04} \text {, } \\
& a_{30}^{\prime}=a_{30}+4 w a_{40}+(t+3 w u) a_{31}+\left(2 u t+2 w u^{2}\right) a_{22} \\
& +\left(3 u^{2} t+u^{3} w\right) a_{13}+4 u^{3} t a_{04}+u a_{21}+u^{2} a_{12}+u^{3} a_{03}, \\
& a_{21}^{\prime}=a_{21}+3 w a_{31}+(2 t+4 u w) a_{22}+\left(6 u t+3 u^{2} w\right) a_{13} \\
& +12 u^{2} t a_{04}+2 u a_{12}+3 u^{2} a_{03} \text {, } \\
& a_{12}^{\prime}=a_{12}+2 w a_{22}+(3 t+3 u w) a_{13}+12 u t a_{04}+3 u a_{03}, \\
& a_{03}^{\prime}=a_{03}+w a_{13}+4 t a_{04} \\
& a_{20}^{\prime}=a_{20}+6 w^{2} a_{40}+\left(3 t w+3 w^{2} u\right) a_{31}+\left(4 u t w+t^{2}+w^{2} u^{2}\right) a_{22} \\
& +\left(3 u t^{2}+3 u^{2} t\right) a_{13}+6 u^{2} t^{2} a_{04}+3 w a_{30}+(t+2 w u) a_{21} \\
& +\left(2 u t+u^{2} w\right) a_{12}+3 u^{2} t a_{03}+u a_{11}+u^{2} a_{02} \text {, } \\
& a_{11}^{\prime}=a_{11}+3 w^{2} a_{31}+\left(4 w t+2 u w^{2}\right) a_{22}+\left(3 t^{2}+6 u t w\right) a_{13} \\
& +12 u t^{2} a_{04}+2 w a_{21}+(2 t+2 u w) a_{12}+6 u t a_{03}+2 u a_{02} \\
& a_{02}^{\prime}=a_{02}+w^{2} a_{22}+3 t w a_{13}+6 t^{2} a_{04}+w a_{12}+3 t a_{03} \text {, } \\
& a_{10}^{\prime}=a_{10}+4 w^{3} a_{40}+\left(3 w^{2} t+w^{3} u\right) a_{31}+\left(2 w t^{2}+2 u t w^{2}\right) a_{22} \\
& +\left(t^{3}+3 u w t^{2}\right) a_{13}+4 u t^{3} a_{04}+3 w^{2} a_{30}+\left(2 w t+1 u w^{2}\right) a_{21} \\
& +\left(t^{2}+2 u t\right) a_{12}+3 t^{2} u a_{03}+2 w a_{20}+(t+w u) a_{11}+2 u t a_{02}+u a_{01} \text {, } \\
& a_{01}^{\prime}=a_{01}+w^{3} a_{31}+2 w^{2} a_{22}+3 t^{2} w a_{13}+4 t^{3} a_{04}+w^{2} a_{21} \\
& +2 t w a_{12}+3 t^{2} a_{03}+w a_{11}+2 t a_{02}
\end{aligned}
$$




$$
\begin{aligned}
a_{00}^{\prime}= & a_{00}+w^{4} a_{40}+w^{3} t a_{31}+w^{2} t^{2} a_{22}+w t^{3} a_{13}+t^{4} a_{04}+w^{3} a_{30} \\
& +w^{2} t a_{21}+t^{2} w a_{12}+t^{3} a_{03}+w^{2} a_{20}+w t a_{11}+t^{2} a_{02}+w a_{10}+t a_{01}
\end{aligned}
$$

If the orbit of $\left(a_{i j}\right)$ has 0 as a limit point, this implies in turn that $a_{04}, a_{13}$, $a_{22}, a_{13}, a_{40}, a_{03}, a_{12}, a_{21}, a_{30}, a_{02}, a_{11}, a_{20}, a_{01}, a_{10}$, and, finally, $a_{\infty 0}$ are zero. Thus, again, because of Theorems 1 and 2, the quotient of $|4 H|$ by $U$ exists in schemes and this quotient is topological.

\section{BIBLIOGRAPHY} \#4273.

1. A. Borel, Linear algebraic groups Benjamin, New York, 1969. MR 40

2. P. Cherenack, Basic objects for an algebraic homotopy theory, Canad. J. Math. 24 (1972), 155-166. MR $45 \# 345$.

3. - The topological nature of algebraic contractions, Comment. Math. Univ. Carolinae 15 (1974), 481-499.

4. J. A. Dieudonné and J. B. Carrell, Invariant theory, old and new, Academic Press, New York and London, 1971. MR 43 \#4828.

5. A. Grothendieck, Éléments de géométrie algébrique. I, Springer-Verlag, Berlin, 1970.

6. D. Mumford, Geometric invariant theory, Ergebnisse der Mathematik und ihrer Grenzgebiete, N. F., Band 34, Springer-Verlag, Berlin; Academic Press, New York, 1965. MR 35 \#5451. bridge, Mass.

7. - Introduction to algebraic geometry, Harvard Univ. Press, Cam-

DEPARTMENT OF MATHEMATICS, UNIVERSITY OF CAPE TOWN, RONDEBOSCH, SOUTH AFRICA 